\title{
COMPETENCY: DEVELOPMENT, INTEGRATION, AND APPLICATION
}

\author{
Steven Moulton \\ HR-Consultant, Action Insight, Inc., Colorado-USA \\ Member, Society of Human Resource Management, Virginia-USA \\ Oki Sunardi \\ HR-Consultant, U-Resource Consulting, Jakarta-Indonesia \\ Member, Society of Human Resource Management, Virginia-USA \\ Gino Ambrosini \\ HR-Consultant, Verdeja \& Asociados, Madrid-Spain \\ Member, Society of Human Resource Management, Virginia-USA
}

\begin{abstract}
:
Many companies and organizations are increasingly focusing on human capital as a competitive advantage in a rapidly changing environment. To achieve business success, companies are expecting their employees to perform at higher levels, to be more customer-responsive, more process-oriented, more involved in shared leadership and more responsible for creating the knowledge that adds value to an organization's distinguishing capabilities. When embarking on the path of selecting and defining competencies, an organization needs to pause for an introspective review. Linking competencies to the organization's purpose, goals and values is the key to positively affect the organization's direction and bottom line. Competencies can be categorized into one of four groups, organization-based, individual-based, technical and behavioral. From a strategic direction approach, the organization that knows and understands its core competencies and capabilities can use them to attain a strategic advantage. In addition, the organization understands that there is a diverse cross section of organizational competencies that are necessary for fulfilling its mission. Successful application of competencies lies in how they are defined. Simplicity and measurability are keys for competencies to be accepted and measured throughout an organization.
\end{abstract}

Keywords: competencies, core competencies, organizational competencies, simplicity and measurability

\section{INTRODUCTION}

Organizations are increasingly focusing on human capital as a competitive advantage in a rapidly changing environment. To achieve business success, companies are expecting their employees to perform at higher levels, to be more customer-responsive, more process-oriented, more involved in shared leadership and more responsible for creating the knowledge that adds value to an organization's distinguishing capabilities.

Adjusting to these challenges has profound implications for human capital management. In particular, human resource professionals are now charged with developing and sustaining methods for: Predicting, in advance, superior performing talent; Aligning individual and team behavior with organization vision and strategy; Integrating performance development systems; Distributing "just in time" learning and development 
opportunities; Building and retaining intellectual capital. To accomplish any of these objectives, HR professionals must be able to help their organizations identify and define required employee competencies. It is now a leading organizational strategy to apply and integrate competencies in all major HR areas, including recruitment, selection, assessment, development, appraisal and rewards.

\section{COMPETENCY DEVELOPMENT}

When embarking on the path of selecting and defining competencies, an organization needs to pause for an introspective review. Linking competencies to the organization's purpose, goals and values is the key to positively affect the organization's direction and bottom line.

Competencies can be linked to both the hard and soft aspects of business. Mission/vision form the basis of determining an organization's strategic direction and its values. Figure 1, demonstrates how core competencies should link to objectives and strategic direction. Also shown is how performance competencies link to the organization's practices and ultimately to its values. Consistent and supportive infrastructure is the key for institutionalizing both sets of competencies across the organization.A great organizational culture does not necessarily mean great results. Likewise, total focus on the quantitative goals, forgetting people, is not a great formula for long-term success. Organizations that seek balance seem to have resilience over the long term. In his book, Good to Great, Jim Collins stated that companies that focus on five areas become far more successful than their competition. Three of these areas fall in the arena of performance competencies and the other two fell into the category of core competencies.

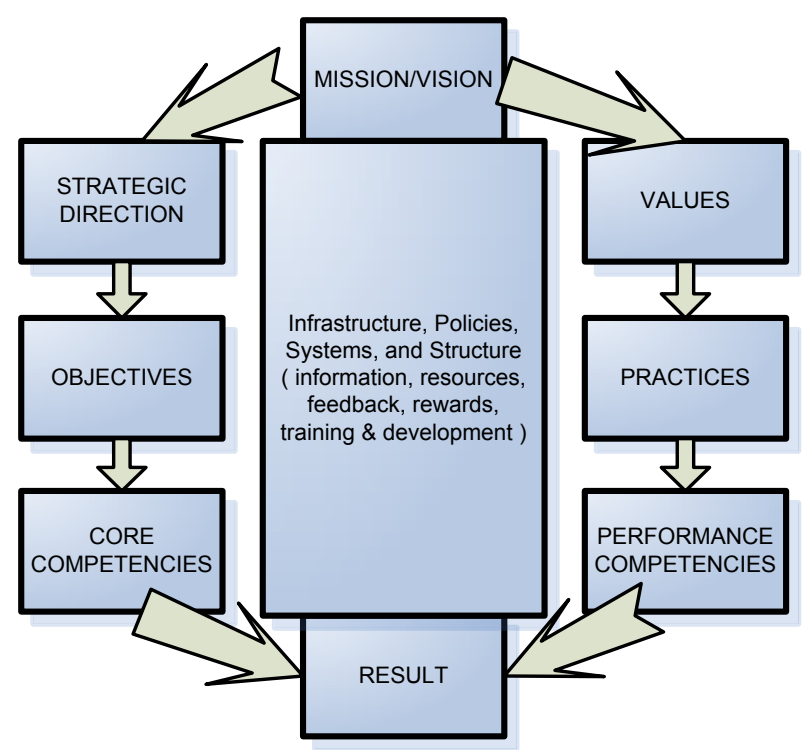

Source: Collins, 2001

Figure 1: Linking Competency

\section{DEFINING COMPETENCIES}

Will competencies be another disappointment for human resource professionals or actually provide a positive impact on the strategic direction and success of an 
organization? The answer will be directly linked to how competencies are created, defined and integrated into an organization's systems, processes and culture.

What is a competency? The word means different things to different people. For an organization, a competency can be defined as the technical capability that differentiates that organization from the competition. On an individual level, a competency can be defined as a group of related knowledge, skills and abilities that influence job performance (Prahalad and Hamel, 1990).

Prahalad and Hamel (1990) also mentioned that competencies can be categorized into one of four groups, organization-based, individual-based, technical and behavioral. Figure 2 illustrates the relationship and difference of each of the types of competencies. It also supports the overall concept of linking the hard and soft aspects of organization planning and development.

\section{STRATEGY/ORGANIZATION CONTENT}

The top half of the diagram reflects the organization's strategy /direction. From a strategic direction approach, the organization that knows and understands its core competencies and capabilities can use them to attain a strategic advantage. In addition, the organization understands that there is a diverse cross section of organizational competencies that are necessary for fulfilling its mission. These core and organization competencies create a context or culture for what and how the organization expects to operate (Prahalad and Hamel, 1990).

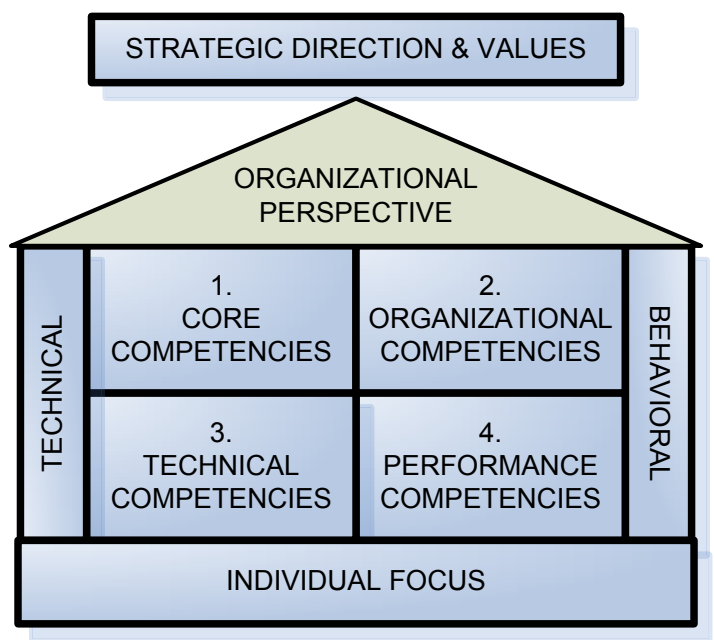

Source: Prahalad and Hamel, 1990

Figure 2. Types of Competencies 


\section{Core Competencies and Capabilities--Quadrant 1}

Core competencies are the distinguishing characteristics of an organization's success. Core competencies are the technical expertise that differentiates an organization from its competition. They are the technologies, strategies, methodologies or processes that create a competitive advantage for an organization. They are not the resources an organization has at its disposal, like money, buildings or equipment.

Prahalad and Hamel (1990) defined the core competency as "...the collective learning in the organization, especially how to coordinate diverse production skills and integrate multiple streams of technologies." Understanding and applying the organization's competencies are significantly different from the traditional application of physical resources. Rather than inventoried and controlled, they need to be shared and cultivated.

\section{Examples:}

\section{Canon}

Using precision mechanics, fine optics and microelectronics to produce such diverse products as cameras, copiers and semiconductor lithographic equipment.

\section{$3 \mathbf{M}$}

Devising various products using substrates, coatings and adhesives has resulted in such diverse products as Post-it ${ }^{\circledR}$ notes, magnetic tape, photographic film, pressure sensitive tapes and coated abrasives.

\section{Organizational Competencies and Capabilities--Quadrant 2}

Organizational competencies and capabilities reflect a list of competencies that describe how the organization expects its employees to accomplish their jobs. The mission, vision, values, culture and core competencies set the tone or work context of the organization.

The context is defined in relation to such values that everyone in the organization must demonstrate, like valuing diversity, quality, innovation or customer service. These values are organizationally focused but not specific enough for application to individual positions.

When a company identifies its core competencies, it is describing WHAT it does best. Organizational competencies and capabilities describe HOW it expects the WHAT to be accomplished. There are usually 15-25 competencies established in order to provide common descriptions of HOW the organization expects its employees to act (Ledford, 1995).

A tapestry of organizational competencies may include such competencies as (Ledford, 1995):

1) Decision-making Risk-taking Develop relationships

2) Problem-solving Analyzing Attention to detail

3) Innovation Resilience Customer service

4) Strategic perspective Team work Leadership 


\section{TACTICAL/INDIVIDUAL CONTENT}

The bottom half of Figure 2 reflects the application of competencies at the individual level. At this level, competencies are important for defining job or work content. Technical and performance competencies are important for success.

When conducting a thorough job analysis, both technical and performance competencies need to be assessed and identified. Experience has shown that most job analysis efforts focus on the technical competency requirements (Ledford, 1995).

Organizations that tend to hire or promote employees for technical skills usually failing for performance issues. Depending on the position, organizations need to consider a balanced approach to assessing new hires, promotions, performance and succession planning.

Ledford (1995) defined these competencies as "demonstrable characteristics of the person, including knowledge, skills, and behaviors that enable performance." In essence, these competencies are how individuals can link what they do and how they do it to the organizational objectives.

\section{Technical/Job Competencies--Quadrant 3}

Technical/job competencies are the specific knowledge, skills and abilities that are applied to accomplishing a specific task, job or function. From an individual's point of view, technical/job competencies, like core competencies, describe WHAT he or she is to do.

Don't confuse technical skills with operational goals. Operational goals are specific to projects or output and technical skills are necessary to make the output happen. For instance, technical competencies might include:

Examples: Completing a balance sheet; Designing a circuit board; Interviewing job Applicants; Operating a computer.

\section{Performance Competencies--Quadrant 4}

From a performance perspective, when an organization identifies its organizational competencies, they are generally intended to apply to all employees. Employees then struggle to translate them into a specific job application. Employees at all levels of the organization struggle because these skills apply to them differently and inconsistently (Ledford, 1995).

Individual performance competencies are more specific than organizational competencies and capabilities. Rather than being defined in a general esoteric sense, they need to be defined in a measurable behavioral context. Also, a variety of competencies need to be defined so as to provide applicability to different positions or jobs to describe $\mathrm{HOW}$ the job is to be done. 
Example: Leadership and tolerance may be important for a human resource executive, but do not apply well to an accountant. The accountant may need performance competence in attention to detail and analytical problem solving.

\section{METHODOLOGY}

Identifying a set of organizational competencies requires a structured methodology. Having a structured methodology for identifying organizational competencies helps maintain both balance and applicability. Organizational competencies can be established as one large grouping of competencies or different sets for different levels of the organization.

Balance and applicability seem to be two areas that are missed when most organizations select organizational competencies. Most competency effort outcomes seem to be focused on executive and management levels of the organization and overlook the competencies required for the rest of the organization.

For example, most organizations tend to identify competencies that are either focused on interpersonal or personal applications. Interpersonal competencies are how people are expected to interact with each other, such as listening or coaching. Personal competencies are how a person internalizes actions, such as self-control or decisiveness.

There is a need to also include competencies that are necessary for both task and interface applications. Task competencies are related to how the job or responsibility gets done, such as problem-solving or persistence. Interface competencies are related to the environment one is working in, including following policies and procedures or ethical/ social responsibility (Ledford, 1995).

There are many approaches that have been used to identify organizational competencies. One approach has been to conduct behavioral or critical event interviews. Selecting the competencies from a list of competencies or bench marking against other companies has also been used.

Whatever the approach, there are seven components necessary to ensure that the outcome will meet the needs of the organization. These seven include: structure, balance, participation, organizational context, job content, linkage and validation (Ledford, 1995).

The approach needs to be structured to identify a balanced set of competencies that would fit the culture of the organization and the four categories of competencies necessary for personal, interpersonal, task and awareness applications. The approach also needs to include a representative participation of the company's population. Many competency lists emphasize management and professional competencies and offer little for the rest of the organization to focus on for alignment.

To create a balanced cross-section of competencies that fit the organizational context, the list may include more than one. Each competency would not apply to each position equally. In other words, at the next level, there must be a job content fit.

How many competencies can someone realistically focus on at a time and do well? How many competencies can realistically be assessed in an interview? Should the jobholder 
focus on the essential competencies or should non-essential competencies require attention as well?

As competencies are developed and implemented in organizations, there are three key factors (Prahalad and Hamel, 1990):

1) The competencies that are to be applied at the individual level need to be linked to the organization level core and organizational competencies.

2) All competencies need to be specific and those that are to be applied in the work environment need to be behaviorally defined.

3) The methodology for identifying the competencies needs to include structure, balance, participation, organizational context, job content, linkage and validation.

Competency-based HR applications represent an integrated framework for maximizing the human capital of an organization. Will competencies be another disappointment for human resource professionals or actually provide a positive impact on the strategic direction and success of and organization? They will have a positive impact, if there is a total and clear integration of core and organizational competencies to the actual work applications and the environment.

The success criteria for driving change related to a competency-based initiative is clear and can be used as a roadmap for designing, sustaining and auditing the competency rollout process. However, practitioners must understand that "dabbling" in competencies almost always does not work and frequently builds resistance. In contrast, competencies make a difference when they are applied consistently in stages over time to achieve systemic change.

\section{APPLYING COMPETENCIES}

Will newly defined competencies be relegated to a binder in a bookcase like so many mission statements are relegated to a wall poster in the front lobby? Or will they become an integrated part of a company's culture? The answer to that question lies in how the competencies are embedded into the organization's systems, particularly the human resource systems and processes.

Covey (1989) emphasizes that "you can't talk your way out of problems you behave yourself into." Defining and applying behavioral competencies can take an organization to a new level of performance.

\section{Trait vs. Behavioral Application}

Successful application of competencies lies in how they are defined. Simplicity and measurability are keys for competencies to be accepted and measured throughout an organization. Defining competencies in esoteric or academic terminology frustrates managers and employees alike.

Some attempts to create and define competencies often end up with esoteric definitions that are not measurable. Although they are clear in intent, the definitions are unusable. 
The behavioral approach for defining tends to make more sense when it comes to applying competencies with real people. By clearly describing how a person can demonstrate a competency, both jobholders and leaders can measure the application. There is also an improved objectivity that improves trust and respect within the organization.

In addition to the benefit of improved objectivity by creating such clear and measurable definitions, it becomes easier to link the competencies you hire people for to how you will measure their job performance.

Linkage to performance management, selection, development and other human resource processes becomes the key to success. How the jobs are analyzed and which competencies are identified as being essential to positions can mean the difference between success and failure. Human resources can add value for line and staff by providing the ability to create integrated structured interviews, performance planning, discussion forms and development programs.

From a practical point of view, let us look at how a competency can be linked from the strategic to the tactical. The following examples describe linkage of a portion of a mission statement to selection, performance, employee development and reward. Note how the underlined portion of the mission statement carries through the competency and its applications.

A phrase from a mission statement:

... a worldwide leader and innovator in flawless production, distribution and delivery of medical products and services.

A phrase from a behavior-based competency:

Performance leadership: Able to influence persons with diverse backgrounds, levels of training, and cultures to adopt new ways of improving work outcomes.

An interview question:

Give me an example of a time when you were able to influence a person to expend a significant effort when learning a new work procedure.

A performance expectation:

Influence coworkers to adopt improved ways of getting work done.

An instructional objective:

On completion of this class, the participant will be able to clearly demonstrate what needs to be done to improve work procedures.

A bonus factor:

You will receive a one-time $\$ 1,000$ bonus if you achieve flawless use of $\mathrm{ABC}$ production equipment by all team members by April 1 .

Each of the examples above becomes part of the processes, language and culture of an organization. There are other issues to consider. For instance, assuring that the process meets validation requirements. 


\section{VALIDATION}

Validation processes are well documented. The three approved processes of validation are: criterion, construct and job content. Of these three, criterion and job content seem to be the most frequently used. In creating an organizational list of competencies with associated linkages of activity statements, interview probes, task statements and other related tools, verifying that there are clear differences and that each set of competency applications clearly link is very important.

\section{CONCLUSION}

By rating the level of importance for each of the activity statements to the position, organizations tend to get to a much more reliable identification of which competencies are essential than by merely selecting competencies from a list. The results can then be linked directly to specific activity statements for selecting interview probes and performance task statements.

Competencies need to be embedded into the organization through the human resource and training systems the organization uses for employee selection, measuring performance, succession planning and employee development.

\section{REFERENCES}

Collins, Jim. Good to Great. New York: Harper Collins Publications, 2001.

Prahalad, C.K. and G. Hamel. "The Core Competence of the Organization," Harvard Business Review. May-June 1990.

Ledford, G.E., "Paying for the Skills, Knowledge, and Competencies of Knowledge Workers," Compensation \& Benefits Review. July-August 1995.

Covey, S.R. The Seven Habits of Highly Effective People. New York: Simon \& Schuster, 1989. 